\title{
Editorial
}

\section{Suicide Prevention Citizenship- Nurturing Future Research Leaders in Suicide Prevention Through Effective Mentorship}

\author{
Jin $\operatorname{Han}^{1}$ and Nicholas Procter ${ }^{2}$
}

'Black Dog Institute, University of New South Wales, Randwick, NSW, Australia

${ }^{2}$ School of Nursing and Midwifery, University of South Australia, Adelaide, SA, Australia

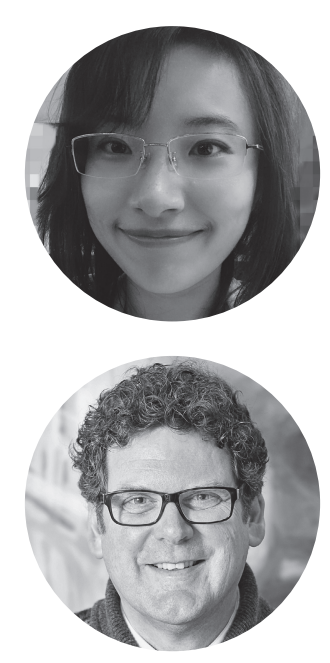

Suicide is one of the biggest health challenges of this century, accounting for $1.4 \%$ of all deaths globally (World Health Organization, 2014). Preventing suicide requires sustained efforts from a range of stakeholders, especially early career researchers (ECRs), to bring in new knowledge and perspectives. However, increasing competition for funding and tenured academic positions is gearing the research milieu toward a more corporate-like "metrics and deliverables"-based environment (Browning, Thompson, \& Dawson, 2016). The evident "Matthew effect" (accumulative advantage) in grant and fellowship applications (Bazeley, 1998) makes the first 3-5 years after PhD a critical period for researchers to establish long-term career success. Without guidance and support from experienced researchers to make timely involvement and connections, talented ECRs may quickly lose their interest and competitiveness in the current research and funding environment (Wyllie, Levett-Jones, DiGiacomo, \& Davidson, 2019), which can threaten the "overall talent-base of academia" (Spence, Buddenbaum, Bice, Welch, \& Carroll, 2018; p. 2). In addition, feelings of frustration and insecurity associated with an uncertain future can reduce ECRs' work productivity and creativity (Aguilera, 2012) and negatively impact personal well-being (Busch \& Ledingham, 2016). Guidance and support are critical for ECRs in suicide prevention research, who are likely to have the aforementioned challenges and elevated psychological distress due to regular exposure to detailed suicide-related information (Chen, Mastarone, \& Denneson, 2019).

Having effective mentorship has been found to help improve ECRs' research productivity and self-efficacy (McRae \& Zimmerman, 2019). Both are important for ECRs, who are likely to face new challenges in personal development, transition, and integration into the scholarly community. Importantly, experienced mentors can also help ECRs construct their professional identity (LaPointe, 2010), which is a profound predictor of long-term persistence in research career (Estrada, Hernandez, \& Schultz, 2018). However, difficulties in building and maintaining effective mentorship have been frequently reported by ECRs (Lalani et al., 2018; Williams, Levine, Malhotra, \& Holtzheimer, 2004).

In this editorial, we provide our insights into how to establish and sustain effective mentorship. We intend to offer an avenue through which discussions on mentorship for ECRs in suicide prevention research can be advanced. To achieve this purpose, we review the current literature on mentorship in mental health research and reflect on our experience in participating the Future Research Leaders Mentoring Program as a mentor (NP) and mentee (JH). This mentorship program is hosted by the Centre for Mental Health at the University of Melbourne in Australia through the Australian Government funded National Leadership in Suicide Prevention Research Project. To our knowledge, it is the first mentorship program designed for building the next generation of suicide prevention researchers. Launched in 2018, this national program aims to "bring emerging Australian suicide prevention researchers with more established counterparts with a view to build the research capacity and leadership skills of Australia's next generation of suicide prevention researchers." We start this editorial by discussing three key factors that contribute to an effective one-to-one mentorship from our experience and a subset of the extant literature. We then compare the structure and effectiveness of the available mentorship programs in mental health research, from where we propose an integrative mentorship model (the Ascending Boomerang Mentorship Model) to sustainably support ECRs in suicide prevention research. We conclude with a few general recommendations for future mentorship programs and the importance 
of driving a collaborative mentorship scheme for ECRs in suicide prevention research.

\section{Three Key Factors in Effective One-to-One Mentorship}

Derived from the root men- meaning to think, mentors are often seen as supportive and protective figures, who provide both professional and personal guidance (Poronsky, 2012). In mentorship, mentors can have multiple roles, including but not limited to being a supporter, role model, collaborator, facilitator, trainer, and communicator (Ambrosetti \& Dekkers, 2010). Traditionally, mentorship happens between an experienced individual and a less experienced person in an either formal (e.g., assigned by the institute) or informal (e.g., connected via personal network) way. Outstanding mentors have been found to demonstrate admirable personal traits such as enthusiasm, compassion, and selflessness (Cho, Ramanan, \& Feldman, 2011). They offer a vision but also tailor it to meet mentees' individual needs. Mentees, on the other hand, need to be honest, open to criticism, proactive, and responsible for the mentorship (Straus, Johnson, Marquez, \& Feldman, 2013; Williams et al., 2004). To help mentees make the best of mentorship, researchers have identified a few characteristics of an effective mentorship, including clear expectations, personal connection, shared values (same chemistry), mutual respect, effective communication, and convergence goals (Keshavan \& Tandon, 2015; Straus et al., 2013). According to our mentorship experience, we distil them to three key factors of a nurturing and reciprocal mentorship, including trust, commitment, and alignment between the mentor and the mentee.

\section{Trust}

Like many other relationships (Sambunjak, Straus, \& Marusic, 2010), we consider trust to be a fundamental requirement for an effective mentoring relationship. Conceptualized by Morgan and Hunt (1994), trust exists when both parties involved in a relationship have confidence in the other's reliability and integrity. Trust is usually built upon personality qualities, such as honesty, consistency, accountability, and fairness (Seppänen, Blomqvist, \& Sundqvist, 2007). In effective mentorship, both mentees and mentors are expected to have these qualities. In addition, mentors are expected to be altruistic, and actually care about mentees' thoughts and interest, or in other words, "prioritise mentees' best interests" (Straus et al., 2013; p. 84). Mentors' motivation to see mentees grow in both academia and personal life is perceived to be the foundation of trust in mentorship. Trust is critical for effective mentorship to flourish as it allows the mentor and the mentee to openly share personal experience in academia and to self-disclose difficult topics such as experiences of disempowerment or feeling undervalued (Alegría, Fukuda, Lapatin Markle, \& NeMoyer, 2019). Trust can also influence the level of commitment by mentors and mentees to mentorship.

\section{Commitment}

It is important for both the mentor and the mentee to make a dedicated commitment to mentorship. Lack of time has been identified as a key barrier to developing effective mentorship (Williams et al., 2004). Keshavan and Tandon (2015) created a mentoring self-appraisal list for researchers to self-check if they are ready to commit time and energy to mentorship. Dedicated commitment from both the mentor and the mentee can be demonstrated by being responsible to the agreed meeting time and tasks. In short, long-term commitment requires a trust-based strong desire to build and keep a valuable relationship, and time availability from both the mentor and the mentee to hold accountable expectations (Watling \& Lingard, 2012).

\section{Alignment}

While trust and commitment are two key factors associated with, especially, the initiation of mentorship, the level of alignment between the mentor and the mentee, or in other words, interpersonal chemistry (Reynolds, Pilkonis, Kupfer, Dunn, \& Pincus, 2007), often impact the sustainability and quality of mentorship. The resonant bond between the mentor and the mentee is dependent on not only similarity of individual experience (e.g., discipline or research interest), expectations, or skills, but also, more importantly, similarity of deep-level personal constructs such as values, beliefs, and norms (Nowell, Norris, Mrklas, \& White, 2017). Resonance is unlikely to be achieved when individuals involved in mentorship have fundamental differences in these deep-level constructs. For example, ECRs who are motivated by a passion for science or social responsibility can feel demotivated if their mentors focus too much on building up the "metrics" to climb a career ladder. One possible approach to reconcile this issue is to integrate one-to-one mentorship into a structured mentorship program where periodic assessments or checking-in with each other can be applied to check the mentorship progress and make necessary adjustments (e.g., find a new mentor if necessary). 


\section{Mentorship Programs in Mental Health Research}

Compared with one-to-one mentorships, mentorship programs are advantageous in providing potentially more structured, comprehensive, and sustainable guidance and support to ECRs (Straus, Graham, Taylor, \& Lockyer, 2008), through offering alternative forms of mentorship (e.g., group or peer mentoring sessions, workshops) or involving additional supporting roles (e.g., grant officers, statisticians). In addition, mentorship programs can be more inclusive to researchers from underrepresented background (e.g., ethnic minorities; Viets et al., 2009), who are often less-active recipients of informal support (Hyers, Syphan, Cochran, \& Brown, 2012). In this section, we overview mentorship programs in mental health research at institutional and cross-institutional (national/ international) levels, briefly discuss program structure and impact, and compare them with the Future Research Leaders Mentoring Program in suicide prevention.

\section{Institution-Based Mentorship Programs}

One of the earliest institution-based mentorship programs reported in mental health research is a formalized mentorship program held by the Department of Psychiatry in the University of Hawaii (Fox, Waldron, Bohnert, Hishinuma, \& Nordquist, 1998). This program adopted traditional one-to-one mentorship model, connecting a senior staff with a new faculty staff. Seven mentees were given an opportunity to choose their mentors from the same department. Group mentoring sessions were purposely excluded from the program owing to concerns about barriers to attendance (e.g., heavy workload and long traveling distance). Specific guidelines on topics (e.g., work and life balance, requirements for promotion) were provided to mentors and mentees before the mentoring sessions. After the first 4 months, assessments were made to check if the mentorship worked for both parties. Satisfied mentors and mentees were then encouraged to meet fortnightly beyond the program. This program was described as a positive experience by both the mentors and the mentees, although no evidence relevant to research productivity or faculty advancement was available.

The Junior Faculty Scholar (JFS; Reynolds et al., 2007) was an institution-based mentorship program designed to facilitate the career development of postdoctoral and junior faculty in mental health research. This program took a collaborative and interdisciplinary approach. It was integrated into the existing postdoctoral, internship, and residency trainings at the hosting institute. Support from the departmental executive board and senior staff was available to extend this mentorship into career development and grant submission. ECRs were given $25 \%$ of their scholar time for mentoring activities, such as weekly research survival skills seminars on career barriers (e.g., poor time management and inadequate grant-preparation skills), and collaborative relationships development in a multidisciplinary research setting. This comprehensive mentorship program unsurprisingly led to a high $\mathrm{K}$ award success rate (100\% in 17 ECRs after two submissions) compared with the $36 \%$ average successful rate over the period of $1997-$ 2003. One of the most important impacts of this program on ECRs, as stated by the authors, was the "consolidation of professional identity" suggested by formal recognition of career directions in ECRs.

In general, institution-based programs are helpful for ECRs to expand professional networks and improve research productivity. Such programs are advantageous in providing a unique chance of being formally integrated into existing activities/training. In this way, mentorship will not create extra workload to ECRs and their mentors. Mentees are also more likely to receive practical advice about career development and navigate the academy with their mentors. As indicated by Law et al. (2014), internal mentors can be more helpful for ECRs to understand internal culture or politics of the work setting. Mentors may become mentees' advocates for promotion and opportunities within the institution. However, institution-based mentorship programs can also lead to restricted collaboration within a small interest group and potentially less open or external communication owing to concerns of confidentiality breach.

\section{Cross-Institutional Mentorship Programs}

Cross-institutional mentorship programs may overcome the aforementioned disadvantages by accessing the broad scholarly community. These programs can help increase ECRs' network opportunities and let them collaborate beyond the usual research teams. Cross-institutional programs can also bring new expertise and knowledge from the external researchers and create a citizenship of nurturing future research leaders in the scholarly community.

The Advanced Research Institute in Geriatric Psychiatry (ARI; Bruce et al., 2011) was a 2-year national mentorship program that aimed to help ECRs in geriatric mental health to develop and obtain their first research grant so as to enable the transition into independent investigators. As one of multicomponent programs to support ECRs in geriatric psychiatry throughout early career stages (Bartels et al., 2010), the ARI targeted the scholars who were in the middle of their early career and ready to submit an independ- 
ent research funding (e.g., NIH R01 funding). The program provided one-to-one mentoring for ECRs to develop a research proposal with their mentor. In the 3-day annual spring retreat, group and personalized opportunities were available for ECRs to advance their research applications via discussions with mentors, peers, and consultants (e.g., statisticians, NIH program officers, senior advisors). The program also involved small group web seminars to facilitate networking of scholars in the relevant fields. In 13 cohorts of ARI scholars $(N=119)$ over the period of 2002 and 2014, 60.4\% of ARI scholars obtained an R01 funding in comparison with $42.0 \%$ of non-ARI scholars (Venegas et al., 2019). ARI scholars were 1.9 times more likely to receive a R01 funding after controlling for the funding year.

The Career Development Institute (CDI) for Psychiatry (Kupfer et al., 2016) was a cross-institutional mentorship program between the University of Pittsburgh and Stanford University. It aimed to provide skills training and support to young psychiatrists and $\mathrm{PhD}$ researchers at critical career transition point (e.g., from postdoctoral research fellow to faculty position). The program lasted for 24 months, consisting of career skills and evaluation (Phase 1), workshops (Phase 2), long-distance mentoring and peer activities (Phase 3), and career progress and program evaluation (Phase 4). The updated program included a 20-month follow-up period to enable continuous mentoring, peer support, and training, recommended by the previous mentees (Kupfer et al., 2009). Mentors in this program were selected from the hosting and external institutions based on their research experience and past successful mentoring experience. Each mentee was paired with one mentor according to their career stage, research interests, and career goals. The program covered a board range of topics, including career planning, budget and project management, grant writing, and psychiatric measurement. The program was continually reviewed to ensure the pairing worked for both parties. Up to 2012, this program benefited 147 ECRs, and generated a support and alumni net in addition to the local network.

\section{Future Research Leaders Mentoring Program}

The Future Research Leaders Mentoring Program is a national mentorship program that aims to build research capacity and leadership skills of the next generation of suicide prevention researchers in Australia. Hosted by the Centre for Mental Health at the University of Melbourne, this program rolled out its first pilot in June 2018, involving 10 pairs of mentees and mentors. It released a following round in September 2018, to meet the increased inter- est and requests from ECRs. In this program, each mentee was matched with a mentor based on their research interest and/or expertise. A welcome e-mail, including an e-booklet of program overview and mentoring resources, was sent to the paired mentor and mentee, to initiate the self-guided mentoring process. Mentors and mentees were also invited to subscribe to a quarterly Suicide Prevention Leaders Network e-Newsletter, introducing upcoming funding and research opportunities, conferences, and recent developments in suicide prevention research. The impact of this program is currently being evaluated by the hosting institute, through collecting feedback from mentors and mentees. An updated mentoring program is expected to be released in early 2020 .

It is noticeable that benefits of cross-institutional/national programs can be limited without enough support from the mentors' and mentees' hosting institutes. It is hence important to create an interconnected and collaborative framework for ECRs mentorship. As Browning et al. (2016; p. 192) stated: "It takes a village to raise an ECR." Collaborative efforts and shared responsibility across institutions and organizations are needed for the development of ECRs in suicide prevention research.

\section{The Ascending Boomerang Mentorship Model in Suicide Prevention Research}

To initiate the collaborative work, we propose a model (see Figure 1) to implement a sustainable mentorship model to support ECRs in suicide prevention research by engaging a range of stakeholders in the process.

The central part of the model is the mentee, bringing to the forefront mentees' benefits in mentorship. The model also emphasizes the role of the mentor, who plays a critical role in enabling and facilitating a personalized approach of mentorship, as the skills required to be a successful suicide prevention researcher can hardly be standardized. We perceive the effective relationship between the mentee and the mentor to be at the core of mentorship, which depends on trust, commitment, and alignment. These three factors can help facilitate an atmosphere of "respect, collegiality, and affirmation" (Vance \& Olson, 1998; p. 5), where development and growth of mentees are more likely to happen. While the static relationship between the mentee and the mentor is shown in Figure 1, in reality, this relationship is dynamic and the roles of mentors and mentees are exchangeable, to pass on knowledge and expertise through generations.

Researchers do not work in a vacuum. Nor do they work in isolation. They need support and inspiration from the 


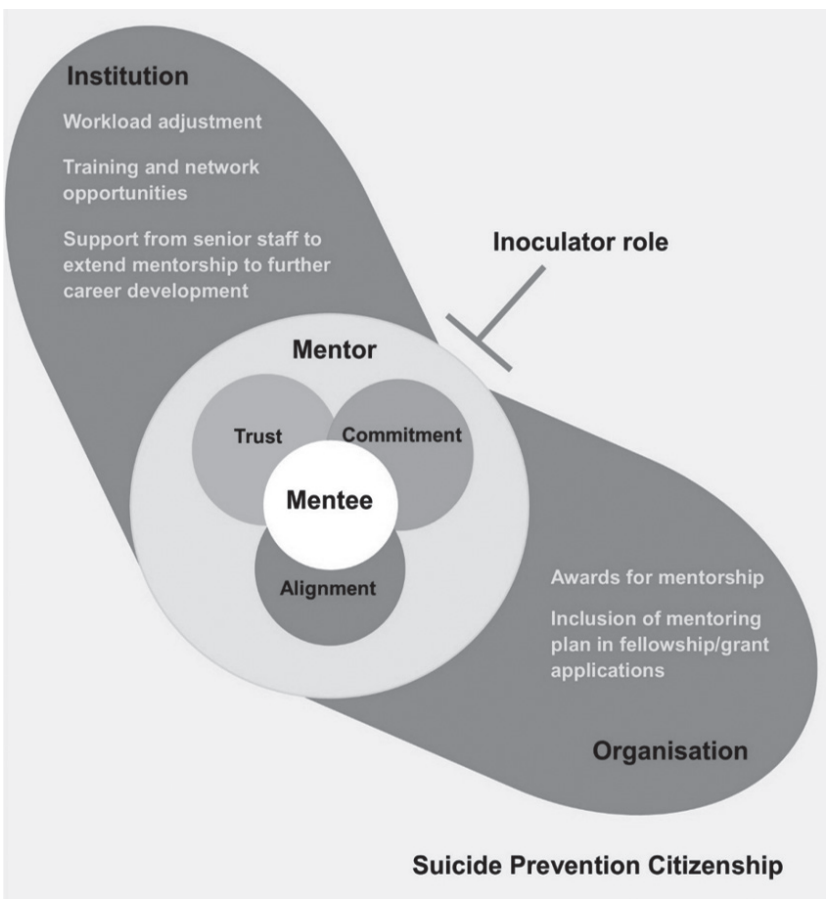

Figure 1. The Ascending Boomerang Mentorship Model in Suicide Prevention Research.

surrounding environment to stimulate and progress their career. In the early-career period, mentees can be especially vulnerable to institutional culture and climate. They may suffer from internal isolation and demotivation if they feel they must deny certain values in order to be successful in career. It is, therefore, important for the host employer to create a supporting environment, and make time and space for mentees and mentors to develop necessary skills and make meaningful connections. We also encourage suicide prevention organizations to create mentoring awards to help protect time for mentoring activities and enhance the recognition of mentorship. An inoculator role is recommended to ensure the psychological safety of mentees and mentors by regularly checking the progress of the mentorship. Lastly, we believe in the notion of mentorship in suicide prevention research as an element of suicide prevention citizenship; whereby mentors and mentees embrace a shared duty and responsibility to co-develop future research leaders in suicide prevention research. We name this the Ascending Boomerang Mentorship Model. The boomerang is a thrown tool used by Indigenous Australians for hunting more than 20,000 years ago. A returning boomerang will always come back to a skilled thrower whichever direction it is thrown. As in mentorship, the ultimate goal of the program is to make our next generation of researchers successful in their field of interest and "come back" as mentors for the benefit of the continued suicide prevention citizenship and as a legacy to mentor others (Law et al., 2014).

\section{Conclusion}

In the busy and highly competitive world of academia, the tradition of altruistic support from senior staff to foster young researchers' transition to independent investigators can easily be at risk of being eroded. The future of suicide prevention research can be compromised if we fail the next generation of upcoming researchers as leaders. Mentorship acts as a critical means of mitigating the challenges and helps to navigate the often-difficult path of academia, easing the transition process of ECRs. In this editorial, we propose a mentorship model for ECRs in suicide prevention based on a rapid literature review and our reflections on participating in the Future Research Leaders Mentoring Program in Australia.

On a personal note, mentorship has been both a rich and prominent experience for both of us. Mentorship is a short-term investment but has great potential to generate long-term benefits for researchers, institutes, and the community. As JH (mentee) reflected:

Mentorship acts a lens to help me see myself for possibilities. Through my mentorship with Professor Procter, I was able to structure my professional identity, which created a precious sense of belongingness and internalising the values of being a good citizen in academia.

As NP (mentor) reflected:

It is what we can accomplish in interaction with each other that helps generate fresh thinking to advance mentee professional development and career goals. I came to this mentorship with the firm intention of doing all I can to ensure the working relationship I have with Dr. Han is successful. Like many of my colleagues, I feel a strong obligation to support the "next generation" of suicide prevention researchers. This aspect is critical and central to effective Suicide Prevention Citizenship.

Being a mentor can be one of the most rewarding things in academia life. Like a Chinese poem describing the role of a mentor, "Sneaking into the tranquil night with the breeze, the rain nurtures each life silently." An effective mentorship serves the future of suicide prevention research silently and gently.

\section{References}

Aguilera, R. J. (2012). Issues on diversity, training, and mentorship of young scientists: An interview. Molecular Biology of the Cell, 23(12), 2237-2239. https://doi.org/10.1091/mbc.E12-03-0208

Alegría, M., Fukuda, M., Lapatin Markle, S., \& NeMoyer, A. (2019). Mentoring future researchers: Advice and considerations. American Journal of Orthopsychiatry, 89(3), 329. https://doi. org/10.1037/ort0000416 
Ambrosetti, A., \& Dekkers, J. (2010). The interconnectedness of the roles of mentors and mentees in pre-service teacher education mentoring relationships. Australian journal of teacher education, 35(6), 3. https://doi.org/10.14221/ajte.2010v35n6.3

Bartels, S. J., Lebowitz, B. D., Reynolds III, C. F., Bruce, M. L., Halpain, M., Faison, W. E., \& Kirwin, P. D. (2010). Programs for developing the pipeline of early-career geriatric mental health researchers: Outcomes and implications for other fields. Academic medicine: journal of the Association of American Medical Colleges, 85(1), 26. https://doi.org/10.1097/ACM.0b013e3181c482cb

Bazeley, P. (1998). Peer review and panel decisions in the assessment of Australian Research Council project grant applicants: What counts in a highly competitive context? Higher Education, 35(4), 435-452.

Browning, L., Thompson, K., \& Dawson, D. (2016). It takes a village to raise an ECR: Organisational strategies for building successful academic research careers. International Journal for Researcher Development, 7(2), 192-197.

Bruce, M. L., Bartels, S. J., Lyness, J. M., Sirey, J. A., Sheline, Y. I., \& Smith, G. (2011). Outcomes of national career development program that promotes the transition to independent scientist. Academic Medicine, 86(9), 1179. https://doi.org/10.1097/ ACM.0b013e3182254399

Busch, R., \& Ledingham, M. (2016). Bothered bloggings and troubled tweets: Constructions of stress and concerns for early-career academics. Paper presented at the 1st International Conference on Advanced Business and Social Sciences, Bali, Indonesia. Retrieved from https://apiar.org.au/?conferencepaper = bothered-bloggings-and-troubled-tweets-construc tions-of-stress-and-concerns-for-early-career-academics

Chen, J. I., Mastarone, G. L., \& Denneson, L. M. (2019). It's not easy - impacts of suicide prevention research on study staff. Crisis, 40(3), 151-156. https://doi.org/10.1027/0227-5910/a000595

Cho, C. S., Ramanan, R. A., \& Feldman, M. D. (2011). Defining the ideal qualities of mentorship: A qualitative analysis of the characteristics of outstanding mentors. The American Journal of Medicine, 124(5), 453-458. https://doi.org/10.1016/j.amjmed.2010.12.007

Estrada, M., Hernandez, P. R., \& Schultz, P. W. (2018). A longitudinal study of how quality mentorship and research experience integrate underrepresented minorities into STEM careers. CBE - Life Sciences Education, 17(1), ar9. https://doi.org/10.1187/ cbe.17-04-0066

Fox, E. C., Waldron, J. A., Bohnert, P., Hishinuma, E. S., \& Nordquist, C. R. (1998). Mentoring new faculty in a department of psychiatry. Academic Psychiatry, 22(2), 98-106. https://doi. org/10.1007/BF03341911

Hyers, L., Syphan, J., Cochran, K., \& Brown, T. (2012). Disparities in the professional development interactions of university faculty as a function of gender and ethnic underrepresentation. The Journal of Faculty Development, 26(1), 18-28.

Keshavan, M. S., \& Tandon, R. (2015). On mentoring and being mentored. Asian Journal of Psychiatry, 16, 84-86. https://doi. org/10.1016/j.a.jp.2015.08.005

Kupfer, D. J., Schatzberg, A. F., Dunn, L. O., Schneider, A. K., Moore, T. L., \& DeRosier, M. (2016). Career development institute with enhanced mentoring: A revisit. Academic Psychiatry, 40(3), 424428. https://doi.org/10.1007/s40596-015-0362-5

Kupfer, D. J., Schatzberg, A. F., Grochocinski, V. J., Dunn, L. O., Kelley, K. A., \& O'Hara, R. M. (2009). The Career Development Institute for Psychiatry: An innovative, longitudinal program for physician-scientists. Academic Psychiatry, 33(4), 313-318. https:// doi.org/10.1176/appi.ap.33.4.313

Lalani, N., Griffith, K. A., Jones, R. D., Spratt, D. E., Croke, J., \& Jagsi, R. (2018). Mentorship experiences of early-career academic radiation oncologists in North America. International Journal of
Radiation Oncology, Biology, Physics, 101(3), 732-740. https:// doi.org/10.1016/j.ijrobp.2018.03.035

LaPointe, K. (2010). Narrating career, positioning identity: Career identity as a narrative practice. Journal of Vocational Behavior, 77(1), 1-9. https://doi.org/10.1016/j.jvb.2010.04.003

Law, A. V., Bottenberg, M. M., Brozick, A. H., Currie, J. D., DiVall, M. V., Haines, S. T., ... Yablonski, E. (2014). A checklist for the development of faculty mentorship programs. American Journal of Pharmaceutical Education, 78(5), 98. https://doi.org/10.5688/ ajpe78598

McRae, M., \& Zimmerman, K. M. (2019). Identifying components of success within health sciences-focused mentoring programs through a review of the literature. American Journal of Pharmaceutical Education, 83(1), 6976.

Morgan, R. M., \& Hunt, S. D. (1994). The commitment-trust theory of relationship marketing. Journal of Marketing, 58(3), 20-38.

Nowell, L., Norris, J. M., Mrklas, K., \& White, D. E. (2017). A literature review of mentorship programs in academic nursing. Journal of Professional Nursing, 33(5), 334-344. https://doi.org/10.1016/j. profnurs.2017.02.007

Poronsky, C. B. (2012). A literature review of mentoring for RN-toFNP transition. Journal of Nursing Education, 51(11), 623-631. https://doi.org/10.3928/01484834-20120914-03

Reynolds, C. F., Pilkonis, P. A., Kupfer, D. J., Dunn, L., \& Pincus, H. A. (2007). Training future generations of mental health researchers: Devising strategies for tough times. Academic Psychiatry, $31(2), 152-159$

Sambunjak, D., Straus, S. E., \& Marusic, A. (2010). A systematic review of qualitative research on the meaning and characteristics of mentoring in academic medicine. Journal of General Internal Medicine, 25(1), 72-78. https://doi.org/10.1007/s11606-0091165-8

Seppänen, R., Blomqvist, K., \& Sundqvist, S. (2007). Measuring inter-organizational trust - a critical review of the empirical research in 1990-2003. Industrial Marketing Management, 36(2), 249-265. https://doi.org/10.1016/j.indmarman.2005.09.003

Spence, J. P., Buddenbaum, J. L., Bice, P. J., Welch, J. L., \& Carroll, A. E. (2018). Independent investigator incubator (I(3)): A comprehensive mentorship program to jumpstart productive research careers for junior faculty. BMC Medical Education, 18(1), 186. https://doi.org/10.1186/s12909-018-1290-3

Straus, S., Johnson, M., Marquez, C., \& Feldman, M. (2013). Characteristics of successful and failed mentoring relationships: A qualitative study across two academic health centers. Academic Medicine, 88(1), 82. https://doi.org/10.1097/ ACM.0b013e31827647a0

Straus, S. E., Graham, I. D., Taylor, M., \& Lockyer, J. (2008). Development of a mentorship strategy: A knowledge translation case study. Journal of Continuing Education in the Health Professions, 28(3), 117-122. https://doi.org/10.1002/chp.179.

Vance, C., \& Olson, R. K. (1998). The mentor connection in nursing. New York, NY: Springer Publishing Company.

Venegas, M., Pepin, R., Bartels, S. J., Lyness, J. M., Sheline, Y. I., Sirey, J. A., ... Bruce, M. L. (2019). Advanced Research Institute (ARI): An effective model for career development and transition to independence. The American Journal of Geriatric Psychiatry, 27(7), 660-663. https://doi.org/10.1016/j.jagp.2018.10.005

Viets, V. L., Baca, C., Verney, S. P., Venner, K., Parker, T., \& Wallerstein, N. (2009). Reducing health disparities through a culturally centered mentorship program for minority faculty: The Southwest Addictions Research Group (SARG) experience. Academic Medicine, 84(8), 1118. https://doi.org/10.1097/ACM.0b013e3181ad$1 \mathrm{cb} 1$

Watling, C. J., \& Lingard, L. (2012). Toward meaningful evaluation of medical trainees: The influence of participants' perceptions 
of the process. Advances in Health Sciences Education, 17(2), 183-194. https://doi.org/10.1007/s10459-010-9223-x

Williams, L. L., Levine, J. B., Malhotra, S., \& Holtzheimer, P. (2004). The good-enough mentoring relationship. Academic Psychiatry, 28(2), 111-115. https://doi.org/10.1176/appi.ap.28.2.111

World Health Organization. (2014). Preventing suicide: A global imperative. Geneva, Switzerland: Author.

Wyllie, A., Levett-Jones, T., DiGiacomo, M., \& Davidson, P. (2019). Exploring the experiences of early career academic nurses as they shape their career journey: A qualitative study. Nurse Education Today, 76, 68-72. https://doi.org/10.1016/j.nedt.2019.01.021

Published online July 16, 2020

\section{Jin Han}

Black Dog Institute

University of New South Wales

Hospital Road

Randwick, NSW 2031

Australia

j.han@blackdog.org.au
Jin Han is a postdoctoral research fellow at the Black Dog Institute of the University of New South Wales. Her research focuses on suicide prevention, with interests including help-seeking in young people, use of technology to reduce suicide risk in the population, and identifying factors that protect against suicide among young people in cross-cultural settings.

Nicholas Procter is Chair of Mental Health Nursing and Leader of UniSA Mental Health and Suicide Prevention Research Group. He contributes to suicide prevention education and research across a range of practice settings. In 2019 he was elected Australia's National Representative to the International Association for Suicide Prevention (IASP) to 2023 